\title{
1. DATA SCIENCE
}

Schon seit Längerem werden z.B. zur Erfassung von Beständen oder zu Buchhaltungszwecken Aufstellungen und Listen angefertigt. Auch natürliche Beobachtungen wie z.B. astronomische Ereignisse wurden so festgehalten. Es ergab sich ein wissenschaftlicher Erkenntnisgewinn, der im Falle Galileo Galileis zur Erschaffung des heliozentrischen Weltbildes führte. Später folgte der Mathematiker Johannes Kepler diesen Spuren und erschuf aus der akribischen Aufzeichnung von Planeten- und Sternenbewegungen ein genaues Planetenmodell unseres Sonnensystems. Albert Einstein baute auf diesem und vielen anderen daten-getriebenen wissenschaftlichen Erkenntnissen auf und leitete schließlich mit seiner Theorie von Energie, Masse und Zeit das Zeitalter der modernen Physik ein.

Seit diesen frühen Tagen ist viel passiert. Anstatt der Männer mit Ferngläsern blicken heute Netzwerke von Teleskopen in die Nacht. Daten, die alleine in diesem Feld erfasst werden, sind für die Menschen jedoch nicht mehr fassbar. Längst hat die maschinelle Verarbeitung solcher Datensätze Einzug gehalten. Aufnahmen von Sternenbildern werden automatisch durchforstet und Auffälligkeiten an menschliche Fachkräfte weitergegeben. Diese wären ohne die Hilfe von Mustererkennung und Methoden der künstlichen Intelligenz längst aufgeschmissen. Dabei ist die Astronomie nur eines der vielen Felder, die sich Methoden der Data Science zu Nutze machen.

Neben der Wissenschaft hat die Industrie endlich das enorme Potenzial, das in der Erfassung der Parameter des Lebens steckt, erkannt. Seien es die Abgrenzung von Werbe-Zielgruppen, die Optimierung von Produktionszyklen, die Analyse der Finanzmärkte, eine schnellere und präzisere Diagnose in der Medizin oder Empfehlungssysteme für den Konsum von Medien, all dies wäre ohne Data Mining nicht möglich. Doch wer denkt, dass diese recht junge Disziplin nicht mehr zu bieten hat, der täuscht sich gewaltig. In Wahrheit stehen wir erst noch ganz am Anfang. IoT-Devices, Quantum Computing und wesentlich mächtigere Techniken des maschinellen Lernens, als wir sie uns heute zu träumen wagen, werden uns auf der Basis von Daten rasant in die Zukunft katapultieren.

\section{INHALT}

\subsection{LERNEN AUS DATEN}

Jana Eschweiler | Durch Datenhoheit zur Smart Factory 23

Michaela Tiedemann | Die Rolle von Data Mining bei Predictive Maintenance in der Automobilbranche 25

Timo Möller | Automatische Textanalyse durch Methoden der künstlichen Intelligenz 27

Stefan Rameseder | Forecasts: Jeder braucht sie, keiner kann sie skalieren $\quad 29$

Eberhard Hechler $\mid$ Auf dem Wege zur Demokratisierung des Maschinellen Lernens 32

\subsection{DIE REVOLUTION DER BRANCHEN}

Robert Jacobi | Ersetzt der Algorithmus den Redakteur?

Alexander Eser | Data Science in der Landwirtschaft: Wie wir mehr Ernte produzieren können

Alexander Eser | Wenn Maschinen Menschen retten: Data Science in der Medizin

Matthias Koeplin | Herausforderungen für Machine Learning in der Abschlussprüfung

Johannes Rupprecht | Praxisbericht: Als Mittelständler selbst KIs trainieren

32

\subsection{BUSINESS INTELLIGENCE}

Dr. Lars Reinkemeyer | Digitale Transformation interner Geschäftsprozesse 44

Markus Gallenberger | Fit for Use: Business-Intelligence und

Analytics brauchen die richtigen Daten zur richtigen Zeit

Dr. Marco Beria | Wie Automatisierung Data Science verändert ... und Data Scientists

Dr. Uwe Müller | Mit Machine Learning Genauigkeit und Effizienz von Prognosen steigern

Tim Hahn | Der E-Commerce ist der beste Einstieg in die Digitalisierung

\subsection{RECHT}

Michael Niederée | Ist die DSGVO das Ende der Data Science?

Dr. Hubert Jäger | Wem gehören die Daten im Connected Car?

Dr. Claudia Schwarz | Gezielter Einsatz eines Patentschutzes für Software als Wettbewerbsinstrument

Dr. Stefan Grotehans | Data Stewardship - die operative Komponente für ein effizientes Data Governance 